\title{
Clinical and molecular study of a pediatric patient with sodium taurocholate cotransporting polypeptide deficiency
}

\author{
MEI DENG ${ }^{1}$, MAN MAO $^{2}$, LI GUO ${ }^{1}$, FENG-PING CHEN ${ }^{2}$, WANG-RONG WEN ${ }^{2}$ and YUAN-ZONG SONG ${ }^{1}$ \\ Departments of ${ }^{1}$ Pediatrics and ${ }^{2}$ Laboratory Science, The First Affiliated Hospital, \\ Jinan University, Guangzhou, Guangdong 510630, P.R. China
}

Received June 6, 2015; Accepted September 6, 2016

DOI: $10.3892 /$ etm.2016.3752

\begin{abstract}
The human solute carrier family 10 member 1 (SLC10A1) gene encodes sodium taurocholate cotransporting polypeptide (NTCP), the principal transporter of conjugated bile salts from the plasma into hepatocytes. Although the function of NTCP has been studied extensively and a number of SLC10A1 variations have been identified in humans, information regarding NTCP deficiency is limited. To date, only one patient with NTCP deficiency has been described; however, in the present study a pediatric patient who experienced intractable and striking hypercholanemia is presented. Analysis of the SLC10A1 gene in the patient revealed a homozygous p.Ser267Phe (c.800C $>$ T) variation, which proved to be a single-nucleotide polymorphism (SNP) in the allele frequency of $4.7 \%$ of healthy controls. This variation involved a conserved amino acid residue on the orthologous alignment that was predicted to be 'disease-causing' by functional analysis using a number of bioinformatic tools. Next generation sequencing was performed; however, no other genetic causes were identified that would affect the bile acid homeostasis in the patient. Moreover, an adult, with the same genotype as the pediatric patient, was identified for the first time as experiencing mild hypercholanemia. The molecular and clinical findings in the present study suggest, for the first time, that there is an association between p.Ser267Phe SNP
\end{abstract}

Correspondence to: Professor Yuan-Zong Song, Department of Pediatrics, The First Affiliated Hospital, Jinan University, 613 Huangpu Da Dao Xi, Guangzhou, Guangdong 501630, P.R. China E-mail: songyuanzong@vip.tom.com

Abbreviations: NTCP, sodium taurocholate cotransporting polypeptide; RFLP, restriction fragment length polymorphism; SNP, single nucleotide polymorphism; NGS, next generation sequencing; MAF, minor allele frequency; SVs, structural variations; ALT, alanine transaminase; AST, aspartate transaminase; GGT, gamma-glutamyl transpeptidase; ALP, alkaline phosphatase; TBA, total bile acids

Key words: sodium taurocholate cotransporting polypeptide, cholestasis, solute carrier family 10 member 1 gene, hypercholanemia, dysfunctional polymorphism and hypercholanemia, and this information may be used to clinically identify NTCP deficiency worldwide.

\section{Introduction}

The human sodium taurocholate cotransporting polypeptide (NTCP) was cloned, localized and functionally characterized in the year 1994 (1). NTCP is encoded by the solute carrier family 10 member 1 (SLC10A1) gene and serves a key role in the enterohepatic circulation of bile salts, acting as the primary transporter of conjugated bile salts from the plasma into hepatocytes $(2,3)$. Although the function of NTCP has been extensively studied and a number of SLC10A1 genetic variations have been identified in humans (4-6), the understanding of NTCP deficiencies remains limited. To date, only one patient with NTCP deficiency has been diagnosed (7); this patient was a pediatric patient with the homozygous and pathogenic p.R252H mutation in the SLC10A1 gene, clinically characterized by marked conjugated hypercholanemia and otherwise unremarkable manifestations. The diagnosis of this child patient with NTCP deficiency clearly established a primary role for NTCP in hepatic bile acid clearance and advances our understanding of normal physiology and the disease (8). However, to the best of our knowledge, no adult patient with NTCP deficiency has been diagnosed yet so far worldwide.

In the present study, the clinical and molecular findings of a second child with NTCP deficiency are described, and an adult with the same genotype and similar, but mild, biochemical alterations are diagnosed as the first adult case of NTCP deficiency..

\section{Materials and methods}

Subjects and ethical approval. A six-month-old male infant, suspected to have NTCP deficiency, and his parents participated in the present study in the First Affiliated Hospital of Jinan University (Guangzhou, China). Clinical data were collected from the child and analyzed. Blood samples from 75 healthy volunteers (with a total of 150 SLC10A1 alleles) were collected for allele frequency analysis of the SLC10A1 variation identified in the patient.

The current study was approved by the Committee for Medical Ethics (The First Affiliated Hospital, Jinan University, 
Table I. Polymerase chain reaction primers and conditions for solute carrier family 10 member 1 gene sequencing.

\begin{tabular}{|c|c|c|c|c|}
\hline Exon & NTCP primer sequence & $\mathrm{AT}\left({ }^{\circ} \mathrm{C}\right)$ & Polymerase & Product (bp) \\
\hline 1 & $\begin{array}{l}\text { Forward 5'-GAAACTAAGGAATCAAGAGCGGAGC-3' } \\
\text { Reverse 5'-CAGGAATTTGAGGTGCTCATTTGG-3' }\end{array}$ & 56 & Taq & 1,248 \\
\hline 2 & $\begin{array}{l}\text { Forward 5'-CTTACTACCTTGTGCGACTTTGAG-3' } \\
\text { Reverse 5'-GGAATTGGATCTTGTTTCTCTCG-3' }\end{array}$ & 56 & Taq & 987 \\
\hline $3-4$ & $\begin{array}{l}\text { Forward 5'-GTACAAAATGTGGTAGCCTATGGAG-3' } \\
\text { Reverse 5'-GTTCTCTGGTCTGTCTTGAGGTTC-3' }\end{array}$ & 56 & LA-Taq & 3,682 \\
\hline 5 & $\begin{array}{l}\text { Forward 5'-CGAAGTTAGAAGTGAAGTGATGATGAAG-3' } \\
\text { Reverse 5'-CTGTGTTTCTCGTTTTGGTGTTGG-3' }\end{array}$ & 58 & Taq & 1,432 \\
\hline
\end{tabular}

AT, annealing temperature; bp, base pairs; NTCP, sodium taurocholate cotransporting polypeptide.

Guangzhou, China). Written informed consent was obtained from the parents of the child and from each healthy volunteer.

SLC10A1 gene analysis. To identify mutations in the SLC10A1 gene of the patient, each of the 5 exons and their flanking sequences, including the 5'- and 3'-untranslated regions and 309 base pairs upstream of the transcriptional start site, were amplified by polymerase chain reaction (PCR) using the primers and polymerases listed in Table I. All PCR amplification was conducted using a Mastercycler nexus PCR instrument (Eppendorf Instrumente GmbH, Hamburg, Germany). SLC25A13 exons 1, 2 and 5 were amplified using a PCR kit (Takara Biotechnology Co., Ltd., Dalian, China) in a $50-\mu 1$ mixture that consisted of $0.25 \mu 1 \mathrm{Taq}(5 \mathrm{U} / \mu \mathrm{l})$, $5 \mu \mathrm{l}$ 10X Buffer ( $\mathrm{Mg}^{2+}$ Plus), $1 \mu \mathrm{l}$ genomic DNA, $4 \mu \mathrm{l} \mathrm{dNTP}$ Mixture $(2.5 \mathrm{mM})$ and $37.75 \mu 1 \mathrm{PCR}$-grade water, besides the relevant primer pair $(1 \mu \mathrm{l}$ of each primer in $20 \mu \mathrm{M})$. For the LA-PCR amplification of exons 3 and 4, the components of the reaction mixture was the same as above but the primer pair and a LA-Taq (Takara Biotechnology Co., Ltd.) instead of the polymerase Taq. The products were purified using a gel extraction kit (Omega Bio-Tek, Inc., Norcross, GA, USA), and analyzed using the Sanger sequencing on a 96-capillary ABI 3730xl DNA Analyzer (Applied Biosystems; Thermo Fisher Scientific, Inc., Foster City, CA, USA) with a BigDye Terminator v 3.1 Cycle Sequencing Kit (Thermo Fisher Scientific, Inc., Waltham, MA, USA), according to the manufacturer's instructions.

PCR-restriction fragment length polymorphism (PCR-RFLP) and variation screening. A novel PCR-RFLP procedure was developed for the present study in order to confirm the genotypes of the patients family and to screen for variation between the 75 healthy volunteers. The nucleotide sequences of the forward and reverse primers used in PCR were 5'-CCA GTTCCCTCTGAGTGTATGTG-3' and 5'-GCAGGCTCA GGTCTAATATTGG-3', respectively (Invitrogen; Thermo Fisher Scientific, Inc.). The $H p h \mathrm{I}$ restriction enzyme was used (Thermo Fisher Scientific, Inc.). The PCR temperature profile was $94^{\circ} \mathrm{C}$ for $5 \mathrm{~min}$, followed by 35 cycles of $94^{\circ} \mathrm{C}$ for $30 \mathrm{sec}, 60^{\circ} \mathrm{C}$ for $40 \mathrm{sec}, 72^{\circ} \mathrm{C}$ for $30 \mathrm{sec}$, then $72^{\circ} \mathrm{C}$ for $10 \mathrm{~min}$. To calculate the variation frequency, the number of alleles bearing the variation in control subjects was divided by 150 then multiplied by 100 .
Alignment of homologous peptides. The peptide identification and amino acid sequences were collected from the orthologue list of the human SLC10A1 gene in the Ensembl Genome Browser (www.ensembl.org). The amino acid sequences of 40 homologous peptides in a diversity of species were aligned using BLAST/BLAT Ensembl software (http://asia. ensembl.org/Multi/Tools/Blast?db=core). Species included primates (human NTCP-1 and apical sodium-dependent bile acid transporter, gorilla, orangutan, olive baboon, gibbon, chimpanzee, macaque, marmoset, vervet African green money and bushbaby), rodents (opossum, rat, mouse and squirrel), laurasiatheria (ferret, armadillo, megabat and microbat), placental mammals (elephant, panda, horse, cow, sheep, alpaca, sloth, tree shrew, pig, dog, cat, shrew and dolphin), sauropsida (duck, chicken, flycatcher, softshell turtle and turkey), fish (cave fish and zebrafish) and invertebrate marine chordate (Ciona intestinalis).

In silico prediction of pathogenicity. PolyPhen-2, MutationTaster and SIFT were used to predict the pathogenicity of the SLC10A1 variation identified in the patient. PolyPhen-2 (http://genetics.bwh.harvard.edu/pph2/) analysis identifies variation as 'probably damaging' if the probability is $>0.85$, and identifies the variation as 'possibly damaging' if the probability is $>0.15$ (9). MutationTaster (http://mutationtaster. org/MutationTaster/index.html) prediction identifies probabilities $\sim 1$ as a 'high security' of the prediction (10). SIFT (http://sift.jcvi.org/www/SIFT_chr_coords_submit. html) identifies the variation as being 'deleterious' if the SIFT score is $<0.05$ (11).

Whole genome sequencing. To eliminate the possibility of other genetic causes resulting in hypercholanemia in the patient, whole genome sequencing was performed using next generation sequencing (NGS). Genomic DNA from the patient was extracted from $2 \mathrm{ml}$ peripheral blood. For quality-control, DNA concentrations were tested with a Nanodrop Spectrophotometer (Thermo Fisher Scientific, Inc.). Following quality-control measurements, the DNA was randomly fragmented into sections of 200-300 base pairs by using a ultrasonicator (Covaris, Inc., Woburn, MA, USA), then fragments of the desired length were purified and separated using gel electrophoresis in a $2 \%$ agarose gel, purified with a QIAquick PCR Purification Kit (QIAGEN, Suzhou, Jiangsu, 
China), added into End Repair Mix (New England Biolabs, Inc., Ipswich, MA, USA), and incubated at $20^{\circ} \mathrm{C}$ for $30 \mathrm{~min}$. The end-repaired DNA was purified with a QIAquick PCR Purification Kit (QIAGEN), then added to an A-Tailing Mix (New England Biolabs, Inc.), and incubated at $37^{\circ} \mathrm{C}$ for $30 \mathrm{~min}$. Then, a ligation reaction was conducted by incubating the purified Adenylate $3^{\prime}$ Ends DNA, Adapter and Ligation Mix (New England Biolabs, Inc.) at $20^{\circ} \mathrm{C}$ for $15 \mathrm{~min}$. Adapter-ligated DNA was selected by running a $2 \%$ agarose gel and purified with a QIAquick Gel Extraction kit (QIAGEN). Following that, several rounds of PCR amplification were performed using a PCR Primer Cocktail and PCR Master Mix (New England Biolabs, Inc.) to enrich the Adapter-ligated DNA fragments. Then the PCR products were selected by running another $2 \%$ agarose gel and purified with a QIAquick Gel Extraction kit (QIAGEN). Adapter ligation and DNA cluster preparation were performed and the DNA fragments were analyzed using the Illumina Hiseq 2000 Sequencing System (Illumina, Inc., San Diego, CA, USA). A total of $95.92 \mathrm{G}$ clean databases were generated for the DNA library that was constructed for the DNA sample. To ensure high quality analysis, the adapter pollutions and the reads which contained $\geq 50 \%$ low quality bases (quality value, $\leq 5$ ) were removed, ensuring that the Q20 base rate of each lane was $>93.77 \%$. The sequencing reads were aligned onto the reference genome sequence using the Short Oligonucleotide Analysis Package aligner/soap2 (http://soap. genomics.org.cn/soapaligner.html; BGI, Shenzhen, China) and single nucleotide polymorphisms (SNPs), InDels and structure variations (SVs) of the sequenced genome were analyzed, as described below.

To annotate and prioritize variation within the gene, common intronic variants were removed following comparison with those in the Single Nucleotide Polymorphism Database and the 1000 Genomes Project (National Center for Biotechnology Information, Bethesda, MD, USA). Synonymous and non-synonymous mutations in exons that were not deleterious following in silico prediction using PolyPhen-2, SIFT or MutationTaster, were also deleted. The variants that were causing abnormal splicing or amino acid changes (including stop-loss and stop-gain variants), in particular those with minor allele frequencies (MAFs) $<1 \%$, were recorded as possible candidates of the causative mutations. In view of the intractable and marked hypercholanemia observed in the patient, a panel of genes that may uniquely affect bile acid homeostasis were assembled for candidate mutation analysis using PubMed, The Online Mendelian Inheritance in Man record and primarily by reference to previous publications (12-16).

In summary, variation pathogenicity was analyzed using in silico methods of prediction using a number online tools. Next, the phenotypic features, including the genetic pattern of the genes involved in harboring pathogenic variations, were compared against the clinical and laboratory findings in the patient. Variations that were identified as causing cholestasis or affecting bile acid homeostasis were considered as being involved in affecting the phenotype of the patient.

\section{Results}

Case report. A 6-month-old male infant was admitted to the First Affiliated Hospital of Jinan University as a result of elevated serum total bile acid (TBA) discovered 4 months previously. At the age of 2 months, the patient underwent a liver function test due to mild jaundice, and it was observed that the levels of TBA and direct bilirubin were elevated. No steatorrhea or acholic stool was observed. After 1 month, the direct bilirubin level recovered gradually; however, the TBA remained elevated for 4 months. The infant was delivered by cesarean section at the gestation age of 38 weeks and 2 days (weight, $3.6 \mathrm{~kg}$; body length, $50 \mathrm{~cm}$ ) due to entanglement of the umbilical cord. The infant's parents were clinically and biochemically healthy with no family history of genetic disease.

The patient underwent a physical examination at 6 months (body weight, $8.5 \mathrm{~kg}$; height, $71 \mathrm{~cm}$; head circumference, $44.5 \mathrm{~cm}$ ) and no dysmorphic appearance or jaundice was observed in the skin and sclera. No stridor, crackles or crepitus was heard with auscultation of the two lungs. The heart sound was normal without any murmurs. The liver and spleen were non-palpable. When picking the infant up, the tone of the body and limbs appeared normal. On biochemical analysis, the levels of TBA reached $492.8 \mu \mathrm{mol} / 1(0-10 \mu \mathrm{mol} / \mathrm{l})$, whereas other indices were normal (Table II). Since citrin deficiency is a common etiology of infant cholestasis in Chinese, genetic analysis of the causative gene, SLC25A13, was performed; however, no mutations were observed, thus eliminating the possibility of citrin deficiency.

During the subsequent 2 years, itchy maculopapular rashes occurred occasionally in the trunk and extremities, and these were attributed to mite dermatitis. This conclusion was reached as serum IgE reached 1,460 IU/ml (0-60), the serum antigens of Dermatophagoides pteronyssinus and Dermatophagoides farinae were strongly positive, and anti-mite measures were able to effectively relieve the severity and frequency of the rash. His TBA reached a surprisingly high level of $737.8 \mu \mathrm{mol} / 1$ at the age of 24.5 months (Table II). Therefore, it was concluded that the pruritus was allergic, not cholestatic, in the patient. Although a low serum level of total $25-\mathrm{OH}$ vitamin D [21.4 $\mathrm{ng} / \mathrm{ml}(30-100)]$ at the age of 2 years and 7 months indicated vitamin D insufficiency, no obvious clinical presentations of fat soluble vitamin malabsorption were observed. The patient's anthropometric indices and social performance developed well without any symptoms or signs of hypotonia, delayed motor milestones and growth retardation on clinical follow-up. However, the hypercholanemia was marked and intractable, regardless of the introduction of cholestyramine at the age of 13 months. Therefore, at 30.5 months, SLC10A1 analysis was performed to evaluate the possibility of NTCP deficiency.

SLC10A1 genotypes and variation frequency. Sanger sequencing of the SLC10A1 gene demonstrated that the patient was a homozygote of the variation c.800C $>$ T (p.Ser267Phe), and that both parents were carriers of the variation. A novel RFLP procedure was developed that confirmed the SLC10A1 genotype of the patient and his parents (Fig. 1). Screening for the p.Ser267Phe variation in the 75 control subjects was performed using the RFLP procedure, revealing 5 heterozygous carriers and 1 homozygote, who was a 30-year-old female without any positive symptoms or signs of NTCP but with a slightly elevated level of TBA [19.3 $\mu \mathrm{mol} / 1(0-10 \mu \mathrm{mol} / \mathrm{l})]$. A $4.7 \%(7 / 150)$ allele frequency within the 75 volunteers indicated that the p.Ser267Phe variation is an SLC10A1 SNP. 
Table II. Biochemical indices over time in a patient with marked hypercholanemia.

\begin{tabular}{|c|c|c|c|c|c|c|c|c|c|c|}
\hline \multirow{2}{*}{$\begin{array}{l}\text { Indices } \\
\text { (reference range) }\end{array}$} & \multicolumn{10}{|c|}{ Months } \\
\hline & 2 & 3 & 4 & 5 & $6^{\mathrm{a}}$ & 8.5 & $13^{\mathrm{b}}$ & 19 & 24.5 & 30.5 \\
\hline ALT (5-40 U/1) & 33 & 30 & 37 & 31 & 32 & 34 & 27 & 24 & 16 & 17 \\
\hline $\operatorname{AST}(5-40 \mathrm{U} / 1)$ & 49 & 32 & 37 & 38 & 37 & 36 & 41 & 37 & 31 & 36 \\
\hline GGT (8-50 U/1) & 220 & 68 & 28 & 11 & 11 & 8 & 9 & 9 & 9 & 9 \\
\hline $\operatorname{ALP}(20-500 \mathrm{U} / 1)$ & 383 & 345 & 317 & 273 & 236 & 332 & 270 & 248 & 254 & 247 \\
\hline Tbil $(2-19 \mu \mathrm{mol} / \mathrm{l})$ & 133.5 & 30.5 & 10.9 & 7.2 & 9.6 & 9.4 & 8.4 & 7.3 & 12.0 & 9.3 \\
\hline Dbil $(0-6 \mu \mathrm{mol} / \mathrm{l})$ & 98.4 & 23.0 & 7.6 & 4.5 & 6.0 & 4.8 & 4.7 & 4.2 & 5.8 & 4.9 \\
\hline Ibil $(2.56-20.9 \mu \mathrm{mol} / \mathrm{l})$ & 35.1 & 7.5 & 3.3 & 2.7 & 3.5 & 4.6 & 3.7 & 3.1 & 6.2 & 4.4 \\
\hline $\mathrm{TBA}(0-10 \mu \mathrm{mol} / \mathrm{l})$ & 221.9 & 151.4 & 431.4 & 251.5 & 492.8 & 567.8 & 653.1 & 494.5 & 737.8 & 405.8 \\
\hline
\end{tabular}

${ }^{a}$ First admission to hospital. ${ }^{b}$ Cholestyramine was introduced. Each TBA value indicates severe hypercholanemia. ALT, alanine transaminase; AST, aspartate transaminase; GGT, gamma-glutamyl transpeptidase; ALP, alkaline phosphatase; Tbil, total bilirubin; Dbil, direct bilirubin; Ibil, indirect bilirubin; TBA, total bile acids.
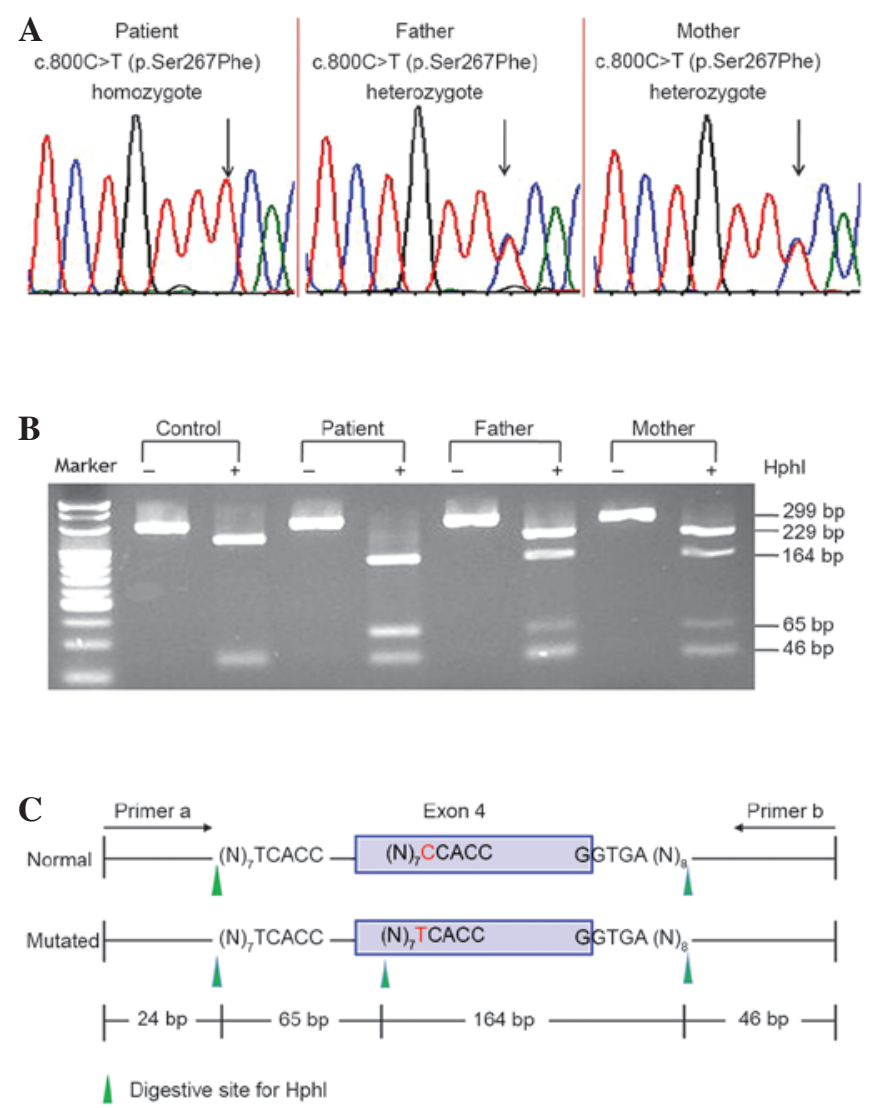

Figure 1 . Solute carrier family 10 member 1 (SLC10A1) genotypes in the family of the pediatric patient and the polymerase chain reaction-restriction fragment length polymorphism (PCR-RFLP) protocol for variation screening. (A) Sanger sequencing revealing that the pediatric patient is a homozygote whose parents are both carriers of the c.800C $>\mathrm{T}$ (p.Ser267Phe) variation. The SLC10A1 genotypes in the family were further confirmed by (B) gel electrophoresis using a newly-developed PCR-RFLP protocol, (C) the schematic diagram of which is illustrated. The variation generated a new digestive site for the restriction enzyme $H p h \mathrm{I}$, producing fragments of 65 and $164 \mathrm{bp}$ from the $229 \mathrm{bp}$ fragment from enzymatic digestion. bp, base pairs.

Bioinformatics. As presented in Fig. 2, the amino acid residue p.267Ser in human NTCP is relatively conserved among aligned homologous peptides from a number of species. SIFT analysis indicated that the c.800C $>\mathrm{T}$ (p.Ser267Phe) variation was predicted to be 'deleterious' (SIFT score, 0.01). Using PolyPhen-2 software, a probability score of 0.959 was calculated, indicating that the variation is 'probably damaging'. Meanwhile, MutationTaster evaluation concluded that the variation is 'disease-causing' (probability value, 0.911).

NGS results. NGS analysis revealed 85 splicing, 80 stop-gain/stop-loss and 10,490 exonic nonsynonymous SNPs, and 586 exonic indels and 76 exonic SVs were detected. Among the NGS analysis results, 239 exonic nonsynonymous SNPs and 4 stop-gains with MAF $<1 \%$ were detected that were 'benign' or 'tolerated' on functional prediction, and not associated with the clinical and biochemical features of the patient.

The bioinformatic tools PolyPhen-2, MutationTaster and SIFT, did not detect any 'deleterious' or 'damaging' variations among the NGS data of the 93 genes which were associated with inherited infantile cholestatic disorders (16). When focusing on the 51 genes that affect bile acid homeostasis uniquely, only 12 exonic nonsynonymous SNPs were detected (Table III). Aside from the p.S267F variation (rs2296651), none of the variations were predicted, clinically or bioinformatically, to be the causative variation of hypercholanemia in the patient. Consequently, the biallelic dysfunctional p.S267F variation in the SLC10A1 gene was concluded to be the unique determinant causing NTCP deficiency in the patient.

\section{Discussion}

Vaz et al (7) first reported a patient with NTCP deficiency, who presented with extremely elevated TBA levels $(\leq 1,500 \mu \mathrm{M}$, reference range, $<16.3 \mu \mathrm{M}$ ) but otherwise a relatively mild clinical phenotype characterized by mild hypotonia, growth retardation and delayed motor milestones. The patient in the present case report presented with intractable and marked hypercholanemia with normal anthropometric development and social performance. The unique disturbance in bile salt homeostasis suggested that NTCP was the most likely affected molecule in the patient. A homologous p.Ser267Phe variation in the SLC10A1 gene was detected using Sanger sequencing, and orthologue alignment and pathogenicity prediction supported 
Table III. Nonsynonymous exonic SNPs detected in genes that may affect bile acid homeostasis.

\begin{tabular}{|c|c|c|c|c|c|c|}
\hline No. & Gene & OMIM ID & Location & dbSNP detected & Amino acid changes & Status \\
\hline 01 & ATP8B1 & 602397 & $18 \mathrm{q} 21.31$ & & & \\
\hline 02 & ABCB 11 & 603201 & $2 q 31.1$ & rs2287622 & p.V444A & Heterozygous \\
\hline 03 & TJP2 & 607709 & $9 q 21.11$ & & & \\
\hline 04 & VPS33B & 608552 & $15 q 26.1$ & rs11073964 & p.G487S & Homozygous \\
\hline 05 & VIPAS39 & 613401 & $14 \mathrm{q} 24.3$ & & & \\
\hline 06 & CCBE1 & 235510 & $18 \mathrm{q} 21.32$ & & & \\
\hline 07 & AMACR & 604489 & $5 \mathrm{p} 13.2$ & rs3195676 & p.V9M & Heterozygous \\
\hline 08 & CYP7A1 & 118455 & $8 \mathrm{q} 12.1$ & & & \\
\hline 09 & AKR1D1 & 604741 & $7 q 33$ & & & \\
\hline 10 & HSD3B7 & 607764 & $16 \mathrm{p} 11.2$ & & & \\
\hline 11 & CYP7B1 & 603711 & $8 \mathrm{q} 12.3$ & & & \\
\hline 12 & CYP27A1 & 606530 & $2 q 35$ & & & \\
\hline 13 & SLC27A5 & 603314 & $19 q 13.43$ & & & \\
\hline 14 & BAAT & 602938 & $9 q 31.1$ & & & \\
\hline 15 & HSD17B4 & 601860 & $5 \mathrm{q} 23.1$ & rs11205 & p.I541V & Homozygous \\
\hline 16 & SLC25A13 & 603859 & $7 q 21.3$ & & & \\
\hline 17 & SERPINA1 & 107400 & $14 q 32.13$ & & & \\
\hline 18 & BCS1L & 603647 & $2 q 35$ & & & \\
\hline 19 & ABCB4 & 171060 & $7 q 21.12$ & & & \\
\hline 20 & CLDN1 & 603718 & $3 \mathrm{q} 28$ & & & \\
\hline 21 & JAG1 & 601920 & 20p12.2 & & & \\
\hline 22 & NOTCH2 & 600275 & 1p12-p11 & & & \\
\hline 23 & CFTR & 602421 & $7 q 31.2$ & & & \\
\hline 24 & Cirhin & 604901 & $16 \mathrm{q} 22.1$ & & & \\
\hline 25 & SLC10A2 (ABST) & 613291 & $13 \mathrm{q} 33.1$ & rs188096 & p.S171A & Heterozygous \\
\hline 26 & EPHX1 & 132810 & $1 q 42.12$ & & & \\
\hline 27 & NTCP (SLC10A1) & 182396 & $14 \mathrm{q} 24.2$ & rs2296651 & p.S267F & Homozygous \\
\hline 28 & ABCC2 (MRP2) & 237500 & $10 \mathrm{q} 24.2$ & rs 17222589 & & \\
\hline 29 & SLCO1B3 & 605495 & $12 \mathrm{p} 12.2$ & & & \\
\hline 30 & SLCO1B1 & 604843 & $12 \mathrm{p} 12.2-\mathrm{p} 12.1$ & & & \\
\hline 21 & MRP6 (ABCC6) & 603234 & $16 \mathrm{p} 13.11$ & & & \\
\hline 32 & ABCG5 & 605459 & $2 \mathrm{p} 21$ & rs6756629 & p.R50C & Heterozygous \\
\hline 33 & ABCG8 & 605460 & $2 \mathrm{p} 21$ & rs6544718 & p.V632A & Homozygous \\
\hline 34 & SLCO2B1 & 604988 & $11 \mathrm{q} 13.4$ & rs2306168 & p.S342F & Heterozygous \\
\hline 35 & SLCO1A2 & 602883 & $12 \mathrm{p} 12.1$ & & & \\
\hline 36 & SLCO4A1 & 612436 & $20 q 13.33$ & & & \\
\hline 37 & $\operatorname{SLC51A}(\mathrm{OST} \alpha)$ & 612084 & $3 q 29$ & & & \\
\hline 38 & SLC51B (OST $\beta)$ & 612085 & $15 q 22$ & & & \\
\hline 39 & TMEM30A & 611028 & 6q14.1 & & & \\
\hline 40 & ТМЕМ30В & 611029 & $14 \mathrm{q} 23.1$ & & & \\
\hline 41 & TMEM30C & 611030 & $3 q 12.1$ & & & \\
\hline 42 & NR1H4 & 603826 & $12 q 23.1$ & & & \\
\hline 43 & CYP8B1 & 602172 & $3 \mathrm{p} 22.1$ & rs9865715 & p.S88P & Homozygous \\
\hline 44 & SLC4A2 & 109280 & $7 \mathrm{q} 36.1$ & & & \\
\hline 45 & SLC10A7 & 611459 & $4 q 31.2$ & & & \\
\hline 46 & SLC22A1 (OCT-1) & 602607 & $6 \mathrm{q} 25.3$ & rs628031 & p.M408V & Homozygous \\
\hline 47 & SLC22A7 (OAT-2) & 604995 & $6 \mathrm{p} 21.1$ & & & \\
\hline 48 & MDR1 (ABCB1) & 171050 & $7 q 21.12$ & & & \\
\hline 49 & MRP3 (ABCC3) & 604323 & $17 q 21.33$ & & & \\
\hline 50 & MRP4 (ABCC4) & 605250 & $13 q 32.1$ & & & \\
\hline 51 & SLC47A1 (MATE-1) & 609832 & $17 \mathrm{p} 11.2$ & & & \\
\hline
\end{tabular}

Empty cells in this table denoted that no nonsynonymous exonic SNPs were detected in the relevant genes. OMIM ID, Online Mendelian Inheritance in Man identification; SNP, single nucleotide polymorphism; dbSNP, The SNP Database. 


\begin{tabular}{|c|c|c|c|c|}
\hline Species & Peptide ID & From & Aminno acid sequence & To \\
\hline Human NTCP-1 & ENSP00000216540 & 248 & RCKRTVSMETGCQNIQLCSTILNVTFPPEVIGPLFFFPLLYMIFQVGEGLLL & 300 \\
\hline Human ASBT & ENSP00000245312 & 252 & YRCRTVAFETGMQNTQLCSTIVQLSFTPEELNVVFTFPLIYSIFQLAFAAIF & 304 \\
\hline Gorilla & ENSGGOP00000022961 & 250 & RCRRTVSMETGCQNVQLCSTILNVAFPPEVIGPLFFFPLLYMIFQLGEGLLL & 302 \\
\hline Orangutan & ENSPPYP00000006762 & 248 & RCRRTVSMETGCQNVQLCSTILNVAFPPEVIGPLFFFPLLYMIFQLGEGLLL & 300 \\
\hline Olive baboon & ENSPANP00000017171 & 248 & RCRRTVSMETGCQNVQLCSTILNVAFPPEVIGPLFFFPLLYMIFQLGEGLLL & 300 \\
\hline Gibbon & ENSNLEP00000002065 & 242 & RCRRTVSMETGCQNVQLCSTILNVAFPPEVIGPLFFFPLLYMIFQLGEGFSX & 294 \\
\hline Chimpanzee & ENSPTRP00000010999 & 248 & RCRRTVSMETGCQNVQLCSTILNVAFPPEVIGPLFFFPLLYMIFQLGEGLLL & 300 \\
\hline Macaque & ENSMMUP00000029493 & 248 & RCRRTVSMETGCQNVQLCSTILNVAFPPEVIGP LFFFPLLYMIFQLGEGLLL & 300 \\
\hline Marmoset & ENSCJAP00000027394 & 246 & RCRRTVSMETGCQNVQLCSTILNGAFPPEVIGPLFFFPLLYMIFQLGEGLLL & 300 \\
\hline Vervet-AGM & ENSCSAP00000009215 & 248 & RCRRTVSMETGCQNVQLCSTILNVAFPPEVIGPLFFFPLLYMIFQLGEGLLL & 298 \\
\hline Bushbaby & ENSOGAP00000013546 & 248 & RCRRTVSMETGCQNVQLCSTILNVTFPPEVIGPLFFFPLLYMIFQLGEGLLL & 00 \\
\hline Elephant & ENSLAFP00000017315 & 251 & QCRRTVSMETGCQNIQLCSTILNVTFPPEVIGPLFFFPL--MIFQIGEGLLF & 300 \\
\hline Panda & ENSAMEP00000015380 & 251 & RCSRTVSMETGCQNVQLCSTILNVTFPPEDIGPLFFFPLLYMIFQLGEGVLL & 301 \\
\hline Horse & ENSECAP00000012659 & 248 & RCRRTVSMETGCQNVQLCSTILNVTFPLEVIGPLFFF PLLYMIFQLGEGLLL & 300 \\
\hline Cow & ENSBTAP00000002446 & 248 & RCRRTVSMETGCQNVQLCSTILNVAFPPEVIGPLFFFPLLYMIFQLGEGLLL & 300 \\
\hline Sheep & ENSOARP00000022773 & 248 & RSKRTVSMETGCQNIQLCSTILNVTFPPEVIGPLFFFPLLYMIFQVGEGLLL & 300 \\
\hline Alpaca & ENSVPAP00000005985 & 248 & RCSRTVSMETGCQNVQLCSTILNVTFPPEVIGPLFFFPLLYMIFQVGEGLLL & 300 \\
\hline Sloth & ENSCHOP00000004848 & 248 & GCKRTVSMETGCQNVQLCSTILNVTFPPEAIGPLFFFPLLYMIFQLGEGLLL & 300 \\
\hline Tree shrew & ENSTBEP00000002346 & 248 & QCSRTVSMETGCQNVQLCSTILNVTFRPEVIGPLFFFPLLYMIFQLGEGLLL & 300 \\
\hline Pig & ENSSSCP00000002511 & 248 & RCSRTVCMETGCQNVQLCSTILNVTFPPEVIGPLFFFPLLYMLFQLGEGLLF & 300 \\
\hline Dog & ENSCAFP00000024409 & 248 & RCSRTVSMETGCQNVQLCSTILNVTFPPEVIGPLFFFPLLYMIFQLGEGVFL & 300 \\
\hline Cat & ENSFCAP00000008204 & 248 & RCRRTVSMETGCQNVQLCSTILNVTFPPQVIGPLFFFPLLYMIFQLGEGVLL & 300 \\
\hline Shrew & ENSSARP00000000978 & 248 & RCRRTVSMETGFQNIQLCSTILNVTFPPEVIGPLFFFPLLYMIFQLAEGLLL & 300 \\
\hline Dolphin & ENSTTRP00000003115 & 252 & HRCRTVALETGMQNTQLCSTIVQLSFTTEELNLTFTFPLIYSIFQLIMAAIF & 300 \\
\hline Opossum & ENSMODP00000014214 & 255 & RCRRTVSMETGCQNIQLCSTILNMAFPPEEIGPLFFLPLLYMIFQLGEGLLL & 304 \\
\hline Rat & ENSRNOP00000007825 & 248 & SCRRTISMETGFQNIQLCSTILNVTFPPEVIGPLFFFPLLYMIFQLAEGLLI & 307 \\
\hline Mouse & ENSMUSP00000093229 & 248 & SCRRTISMETGFQNVQLCSTILNVTFPPEVIGPLFFFPLLYMIFQLAEGLLF & 300 \\
\hline Squirrel & ENSSTOP00000003472 & 248 & RCRRTISMETGFQNIQLCSTILNVTFPPEVIGPLFFFPLLYMIFQLGEGLLF & 300 \\
\hline Ferret & ENSMPUP00000007411 & 248 & RCSRTVSMETGCQNVQLCSTILNVTFRPEDIGPLFFFPLLYMIFQLGEGVLL & 300 \\
\hline Armadillo & ENSDNOP00000004782 & 248 & RGRRTVSMETGCQNVQLCATILNVTFPPEVIGPLFFFPLLYMIFQLGEGLFL & \\
\hline Megabat & ENSPVAP00000009265 & 248 & XXXCRHTVETGCQNIQLCSTILNVTFPPEVIGPL-FFPLLYMICQVAEGFLL & 300 \\
\hline Microbat & ENSMLUP00000010888 & 251 & RCRRTVSMETGCQNIQLCSTILNVTFPPEVIGPLFFFPLLYMICQLGEGLLL & 303 \\
\hline Duck & ENSAPLP00000014650 & 256 & QRSRTQVLQTPLKNVQLCSTILKVAFAPEVIGPLYFFPLLYLMFQL--.-- & 308 \\
\hline Chicken & ENSGALP00000015323 & 248 & RCRQTVCMATGCQNVQLCSTILTVAFANEIIGPLYLFPLLYLIFQLGEGLLL & 300 \\
\hline & ENSFALP00000005168 & 248 & RCRRTVCMETGGQNAQLCSAILKVTFAPEIIGPLYFFPLLYLLFQLGEGFLL & 300 \\
\hline Softshell turtle & ENSPSIP00000000442 & 247 & RCRRTVSMETGCQNVQLCSTILNVAFPPEVIGPLFFFPLLYMIFQLGEGLLL & 299 \\
\hline Turkey & ENSMGAP00000012582 & 244 & HCRRTVSLETGCQNVQLCTAILKLTFPPQLVGGMYMFPLLYALFQAAEAGLF & 296 \\
\hline Cave fish & ENSAMXP00000012978 & 258 & QCKRTIAVETGCQNIQLCSTILKVAFRPEDIGPLYLFPLIYIFQGGEALLF & 312 \\
\hline Zebrafish & ENSDARP00000113054 & 256 & PCRRTVSMEVGCQNIQLCTTILKVAFPAEIIGRLYFFPVIYIVFQIVEALIF & 308 \\
\hline Ciona intestinalis & ENSCINP00000008688 & 224 & NSRRTVAIETGCQNSQLSSTILKMAFANEVMGAYFLFP-LYALFQGLEGLAM & 275 \\
\hline
\end{tabular}

Figure 2. Alignment of homologous peptides in a diversity of species. With the exception of armadillo, turkey and zebrafish, all the remaining 37 species, including primates, rodents, laurasiatheria, placental mammals, sauropsida, fish and marine chordate, have the same serine residue as highlighted in green This indicates that the p.Ser267Phe variation affected a number of conserved amino acid residues among species. NTCP, sodium taurocholate cotransporting polypeptide; ASBT, apical sodium-dependent bile acid transporter.

the disease-causing nature of this variation. In addition, the p.Ser267Phe variant was reported to exhibit a near complete loss of function of bile acid uptake, causing NTCP to lose its taurocholate transporting activity $(5,17)$.

The experimental findings in the present study were similar to the functional analysis of the causative mutation p.Arg252His in the first patient that was reported to have NTCP deficiency, who was a homozygote of the causative mutation p.Arg252His (7). The p.Ser267Phe and p.Arg252His variations demonstrated similarly strong experimental and bioinformatic pathogenicity $(5,7,17)$; using various in silico tools, the p.Arg252His mutation displayed a disease-causing nature with a score of 1.000 using PolyPhen-2, 0.9994 using MutationTaster and 0 using SIFT analysis. These results are similar to the functional predictions of the p.Ser267Phe SNP in the current study. Together, this evidences supports the diagnosis of NTCP deficiency in the patient in the present study.

As the genetic determinants of hypercholanemia are complex (16), whole genome sequencing was performed in the present study in order to evaluate the possible involvement of other genes, the collective testing of which was prohibitively time-consuming and cost-expensive. The sequencing revealed that no other genetic mutations were causing perturbations of bile acid metabolism other than the SLC10A1 gene, and this further supports the diagnosis of NTCP deficiency in the patient. To the best of our knowledge, this is the second report of a patient with NTCP deficiency in the world.

The allele frequency of the p.Ser267Phe variation was calculated to be $4.7 \%$ in control subjects. This is consistent with previous studies which demonstrated that this polymorphism is common in East Asian countries including China and Vietnam, but not in European American, African American or Hispanic countries $(4-6,18)$. NTCP is the functional receptor for human hepatitis B and D viruses (19). The p.Ser267Phe variation experimentally abrogates the ability of NTCP to support HBV infections in cell culture (17), and is associated with resistance to chronic hepatitis B in humans (18). This supports the concept that p.Ser267Phe SNP severely impairs the function of NTCP, and suggests that its high allele frequency may be a result of positive selection in Eastern Asia, where hepatitis B is more prevalent than in European American, African American or Hispanic countries (18). 
Besides the NTCP-deficient biallelic p.Ser267Phe in the child patient in the present study, an adult homologous for the same dysfunctional SLC10A1 SNP was identified in the present study, who presented with slight hypercholanemia without other clinical or laboratory anomalies. The mechanism underlying the more prominent hypercholanemia in the infant, in comparison with the adult with the same SLC10A1 genotype, remains unknown. It is likely that the adult homozygote of the p.Ser267Phe SNP had, or lacked, a number of other genomic SNPs that were analyzed in the child (Table III). Another possible explanation is that the impaired NTCP function in the adult may have been compensated for by other transporters with the ability to uptake bile acids from the plasma, such as Organic Anion Transporting Polypeptide (OATP) 1B1 and OATP1B3 in the basolateral membrane of hepatocytes (20), and the type II form of microsomal epoxide hydrolase (21).

In addition to the prominent hypercholanemia, the elevated serum levels of ALT, AST, TBil and DBil in the child (Table II) suggested the presence of transient cholestasis in early infancy. The increased levels of GGT within the first 3 months after birth may have been partially attributed to cholestasis, although GGT activity is typically higher in infants than in adults (22). As the child was delivered by cesarean section due to entanglement of the umbilical cord, hypoxic ischemia of the liver may have been a likely cause for the transient elevation of the cholestatic indices. Another possibility is that the transient cholestatic liver disease experienced in early infancy may be a phenotypic feature of NTCP deficiency in the child. However, further research is required to determine whether liver disease is common in pediatric patients with NTCP deficiency.

In summary, the current study presents a pediatric patient who demonstrated prominent hypercholanemia with otherwise unremarkable presentations. SLC10A1 gene analysis revealed the presence of a p.Ser267Phe homozygote that is bioinformatically analyzed as a dysfunctional SNP that prevents the functioning of NTCP. Combined with the results from an adult with mild hypercholanemia, the diagnosis of the child with NTCP deficiency suggests, for the first time, that there is an association between the dysfunctional SNP and hypercholanemia. To the best of our knowledge, this is the second clinical description on NTCP deficiency worldwide.

\section{Acknowledgements}

The authors thank Dr Qun-Zhou Zhang for the revision of the manuscript. The present study was supported by the National Natural Science Foundation of China (grant nos. 81270957 and 81570793) and the Cultivation Foundation for Scientific Research (grant no. 2014208) approved by the First Affiliated Hospital, Jinan University .

\section{References}

1. Hagenbuch B and Meier PJ: Molecular cloning, chromosomal localization, and functional characterization of a human liver $\mathrm{Na}^{+} /$bile acid cotransporter. J Clin Invest 93: 1326-1331, 1994.

2. Hagenbuch B and Dawson P: The sodium bile salt cotransport family SLC10. Pflugers Arch 447: 566-570, 2004.
3. Anwer MS and Stieger B: Sodium-dependent bile salt transporters of the SLC10A transporter family: More than solute transporters. Pflugers Arch 46: 77-89, 2014.

4. Saito S, Iida A, Sekine A, Ogawa C, Kawauchi S, Higuchi S and Nakamura Y: Catalog of 238 variations among six human genes encoding solute carriers (hSLCs) in the Japanese population. J Hum Genet 47: 576-584, 2002.

5. Ho RH, Leake BF, Roberts RL, Lee W and Kim BB: Ethnicity-dependent polymorphism in $\mathrm{Na}^{+}$-taurocholate cotransporting polypeptide (SLC10A1) reveals a domain critical for bile acid substrate recognition. J Biol Chem 279: 7213-7222, 2004.

6. Pan W, Song IS, Shin HJ, Kim MH, Choi YL, Lim SJ, Kim WY, Lee SS and Shin JG: Genetic polymorphisms in $\mathrm{Na}^{+}$-taurocholate co-transporting polypeptide (NTCP) and ileal apical sodium-dependent bile acid transporter (ASBT) and ethnic comparisons of functional variants of NTCP among Asian populations. Xenobiotica 41: 501-510, 2011.

7. Vaz FM, Paulusma CC, Huidekoper H, de Ru M, Lim C, Koster J, Ho-Mok K, Bootsma AH, Groen AK, Schaap FG, et al: Sodium taurocholate cotransporting polypeptide (SLC10A1) deficiency: Conjugated hypercholanemia without a clear clinical phenotype. Hepatology 61: 260-267, 2015.

8. Karpen SJ and Dawson PA: Not all (bile acids) who wander are lost: the first report of a patient with an isolated NTCP defect. Hepatology 61: 24-27, 2015.

9. Adzhubei IA, Schmidt S, Peshkin L, Ramensky VE, Gerasimova A, Bork P, Kondrashov AS and Sunyaev SR: A method and server for predicting damaging missense mutations. Nat Methods 7: 248-249, 2010.

10. Schwarz JM, Cooper DN, Schuelke M and Seelow D: MutationTaster2: Mutation prediction for the deep-sequencing age. Nat Methods 11: 361-362, 2014.

11. Kumar P, Henikoff S and Ng PC: Predicting the effects of coding non-synonymous variants on protein function using the SIFT algorithm. Nat Protoc 4: 1073-1081, 2009.

12. Boyer JL: Bile formation and secretion. Compr Physiol 3: 1035-1078, 2013.

13. Hirschfield GM: Genetic determinants of cholestasis. Clin Liver Dis 17: 147-159, 2013.

14. Marschall HU and Beuers U: When bile acids don't get amidated. Gastroenterol 144: 870-873, 2013.

15. Sambrotta M, Strautnieks S, Papouli E, Rushton P, Clark BE, Parry DA, Logan CV, Newbury LJ, Kamath BM, Ling S, et al: Mutations in TJP2 cause progressive cholestatic liver disease. Nat Genet 46: 326-328, 2014.

16. Herbst SM, Schirmer S, Posovszky C, Jochum F, Rödl T, Schroeder JA, Barth TF, Hehr U, Melter M and Vermehren J: Taking the next step forward - Diagnosing inherited infantile cholestatic disorders with next generation sequencing. Mol Cell Probes 9: 291-29, 2015.

17. Yan H, Peng B, Liu Y, Xu G, He W, Ren B, Jing Z, Sui J and Li W: Viral entry of hepatitis B and D viruses and bile salts transportation share common molecular determinants on sodium taurocholate cotransporting polypeptide. J Virol 88: 3273-3284, 2014.

18. Peng L, Zhao Q, Li Q, Li M, Li C, Xu T, Jing X, Zhu X, Wang Y, et al: The p.Ser267Phe variant in SLC10A1 is associated with resistance to chronic hepatitis B. Hepatology 61: 1251-1260, 2015.

19. Yan H, Zhong G, Xu G, He W, Jing Z, Gao Z, Huang Y, Qi Y, Peng B, Wang H, et al: Sodium taurocholate cotransporting polypeptide is a functional receptor for human hepatitis B and D virus. eLife 1: e00049, 2012.

20. Hagenbuch B and Meier PJ: The superfamily of organic anion transporting polypeptides. Biochim Biophys Acta 1609: 1-18, 2003.

21. Zhu QS, Xing W, Qian B, von Dippe P, Shneider BL, Fox VL and Levy D: Inhibition of human m-epoxide hydrolase gene expression in a case of hypercholanemia. Biochim Biophys Acta 1638: 208-216, 2003.

22. Cabrera-Abreu JC and Green A: Gamma-glutamyltransferase: Value of its measurement in paediatrics. Ann Clin Biochem 39: 22-25, 2002. 